\title{
Análisis de la meiosis y viabilidad del polen en morfotipos intraespecíficos de Cuscuta parodiana [Cuscutaceae]
}

\author{
Analysis of meiosis and pollen viability in intraspecific morphotypes \\ of Cuscuta parodiana [Cuscutaceae)
}

D.o.I.: doi.org/10.30550/j.lil/2018.55.1/1

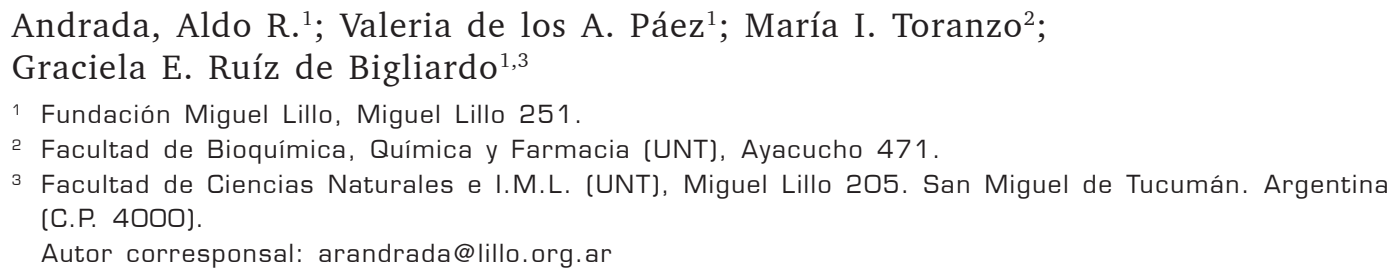

> Resumen - En la familia Cuscutaceae, el género Cuscuta está representado por plantas holoparásitas con distribución cosmopolita. El taxón incluye tres subgéneros: Cuscuta, Monogyna y Grammica; este último muy representado en América. En este trabajo se exponen la morfología floral, estudios meióticos y viabilidad del polen de ejemplares colectados en cuatro (4) poblaciones de Cuscuta parodiana del subgénero Grammica del Noroeste Argentino. El análisis de la morfología floral permitió diferenciar 4 morfotipos en base a los caracteres de la corola y el gineceo. De las entidades estudiadas tres fueron tetraploides ( $n=30$ ) y uno de ellos diploide $(n=15)$. Durante la meiosis se han observado configuraciones trivalentes y tetravalentes, puentes de cromatina, cromosomas fuera de placa metafásica, citomixis y cuerpos redondeados extranucleares.

Palabras clave: Cuscuta parodiana, morfotipos, poliploidía, viabilidad de polen.

Abstract - In family Cuscutaceae the Cuscuta genus comprises holo-parasitic plants that have cosmopolitan distribution. The genus includes three subgenera: Cuscuta, Monogyna and Grammica; the last one is widely distributed in the American continent. In this work, flower morphology, meiotic studies and pollen viability of 4 natural populations of Cuscuta parodiana subgenus Grammica are presented. The flower morphology allow us to differentiate 4 morphotypes through corolla and gynoecium traits. Three entities analyzed were tetraploids $(n=30)$, and one was of them diploid $(n=15)$. Trivalent and tetravalent chromosomal configurations, chromatin bridges, chromosomes outside of metaphasic plate, cytomixis and extranuclear spherical bodies were observed during meiosis.

Keywords: Cuscuta parodiana, morphotypes, polyploidy, pollen viability.

\footnotetext{
> Ref. bibliográfica: Andrada, A. R., Páez, V. de los A., Toranzo, M. I., Ruiz de Bigliardo, G. E. (2018). Análisis de la meiosis y viabilidad del polen en morfotipos intraespecíficos de Cuscuta parodiana (Cuscutaceae). Lilloa 55 (1): 3-15.

> Recibido: 02/08/17 - Aceptado: 15/05/18

$>$ URL de la revista: http://lilloa.lillo.org.ar

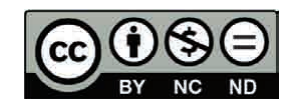

OPEN 0 ACCESS

> Algunos derechos reservados. Esta obra está bajo una Licencia Creative Commons Atribución - No Comercial - Sin Obra Derivada 4.0 Internacional.
} 


\section{INTRODUCCIÓN}

La familia Cuscutaceae está integrada por especies holoparásitas, que se distribuyen en el viejo y nuevo mundo, y comprende entre 165-175 especies descriptas hasta el momento (Stefanovic, Kuzmina, Costea, 2007). Los caracteres anatómicos y morfológicos le permitieron a Yuncker (1932) reconocer tres subgéneros ampliamente distribuidos, Cuscuta Engelmann, Grammica Yuncker y Monogyna Engelmann ex Yunck. Las especies del subgénero Grammica están presentes en todos los continentes y el mayor número se registra en América. Aquellas pertenecientes a los subgéneros Cuscuta y Monogyna se distribuyen en Europa, Asia, África y América del Norte.

Los estudios citológicos realizados en el género Cuscuta demuestran la presencia de cromosomas monocéntricos en Monogyna y Grammica, mientras que holocéntricos en el subgénero Cuscuta (Fogelberg, 1938; Kaul y Bhan, 1977; Pazy, 1997; Pazy y Plitmann, 1994, 1995, 2002; García, 2001; García y Castroviejo, 2003). Los antecedentes de los números cromosómicos en el género se ubican en el rango desde $2 \mathrm{n}=8$ a $2 \mathrm{n}=60$ cromosomas, los números más bajos pertenecen al subgénero Cuscuta y los más altos al subgénero Grammica (Pazy y Plitmann, 1995). Se presentan complejos poliploides, tal es el caso de Cuscuta reflexa Roxb. (subgénero Monogyna) con recuentos cromosómicos variables de $2 \mathrm{n}=14,28,30,32,42$, 48 (Sharma y Chatterji, 1957; Finn, 1937; Kaul y Bhan, 1974; Vasudevan, 1975; Kaul y Bhan, 1977; Vir Jee y Kachroo, 1985). Los dos números básicos más difundidos en el género Cuscuta son $\mathrm{x}=14 \mathrm{y} \mathrm{x}=15 \mathrm{y}$ habrían surgido por una euploidía secundaria, producto del probable número ancestral $\mathrm{x}=7$ (Lewis, 1980). Los reordenamientos cromosómicos debido a disploidía descendente y ascendente, junto a los de poliploidía, podrían ser los responsables de la extensa variación en los números cromosómicos encontrados en la familia (García y Castroviejo, 2003).

Cuscuta parodiana Yuncker está incluida en el subgénero Grammica, es endémica de
Argentina y ocupa las provincias de Jujuy, Salta y Tucumán desde los 500 a 2000 m snm (Zuloaga y Morrone, 1999). Es una planta herbácea, parásita y arbustiva en las regiones montañosas húmedas (Huzinker, 1947). Este último autor reconoce en $C$. parodiana un gran polimorfismo por el que distingue, dos variedades relacionadas con el tamaño y morfología de las piezas florales, C. parodiana var. typica y C. parodiana var. tucumana Yunck. (Hunz.). Recientemente, estudios meióticos realizados por Páez, Andrada, Lozzia, Toranzo (2011) mencionaron para $C$. parodiana $\mathrm{n}=15$ para una población de Tafí del Valle (Tucumán, Argentina).

Durante las colecciones de ejemplares de C. parodiana en Noroeste Argentino (NOA) se observaron morfotipos que exhibieron variaciones en la forma y el tamaño de sus flores cuyos caracteres no se correspondían con las variedades establecidas por Huzinker (1947).

El objetivo de este trabajo es dar a conocer la variabilidad de la morfología floral, los números cromosómicos, el comportamiento meiótico y la viabilidad de los granos de polen de cuatro morfotipos de $C$. parodiana del NOA.

\section{MATERIALES Y MÉTODOS \\ MATERIAL ESTUDIADO}

El material recolectado (Fig.1-2) se encuentra depositado en el herbario Fanerogámico de la Fundación Miguel Lillo (LIL) y proviene de las regiones del Noroeste Argentino (NOA) que abajo se detallan.

ARGENTINA. Prov. Jujuy, Dpto. Tumbaya, Loc. Bárcena, $24^{\circ} 01^{\prime} 49.8$ S, 65²6'21.7” W, 1675 m snm, 15-IV-2009, Cristóbal y Lozzia s/n (LIL 610029). Prov. Tucumán, Dpto. Burruyacú, Loc. Sa. Medina, 26²6'39.8 S, 6502'42.48 W, $1250 \mathrm{~m} \mathrm{snm}, 18-\mathrm{IV}-2008$, Andrada y Páez s/n (LIL 610028); Dpto. Trancas. Loc. Hualinchay, 24¹8'54.7” S, 65³7'21.7" W, 1980 m snm, 17-III-2008, Andrada y Páez s/n ( LIL 610027, 610030).

\section{ESTUDIOS MORFOLÓGICOS}

Para el análisis de la morfología floral se tomaron 15 flores pertenecientes a 3 plan- 
tas diferentes de cada población, las que se recolectaron al azar inmediatamente después de la antesis. Para el caso se fijaron en solución de alcohol etílico-ácido láctico 5:1 (Fernández, 1973). Asimismo, se analizaron igual número de flores extraídas de material herborizado. Se tomaron los siguientes parámetros cualitativos: 1) forma de la corola, 2) forma y tamaño relativo del ovario y estilo, 3) forma de las escamas y su ubicación respecto a la corola (Tabla 1). Las observaciones se llevaron a cabo en microscopio óptico compuesto (lupa) Zeiss Stemi 2000-C.

Análisis de la meiosis: Los botones florales jóvenes se fijaron en solución de Farmer y se conservaron en etanol $70^{\circ}$ a $-4^{\circ} \mathrm{C}$.
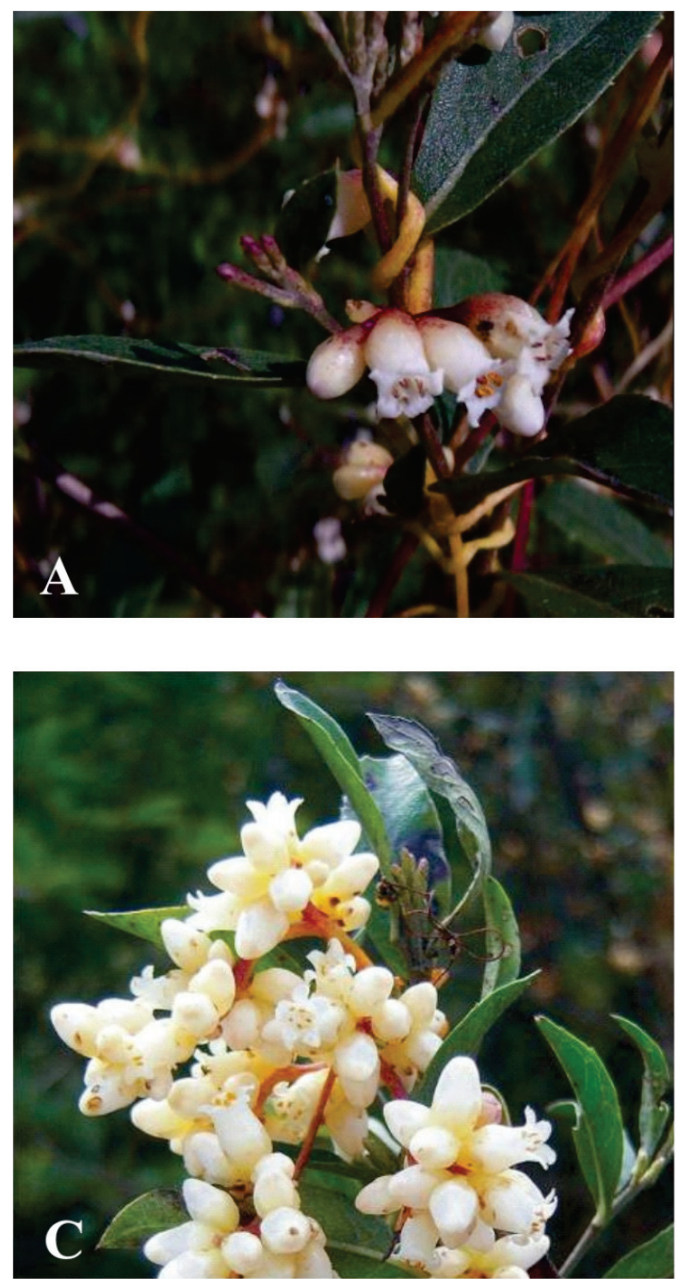

El protocolo de coloración y montaje de las preparaciones microscópicas incluye una hidrólisis previa en $\mathrm{HCl} 1 \mathrm{~N}$ a $60^{\circ} \mathrm{C}$, durante 20 minutos y tinción con una gota de hematoxilina propiónica al $2 \%$ con cristales de citrato férrico. Los recuentos cromosómicos se efectuaron en 7 células madre del polen en diacinesis o metafase I. Se analizaron 50 células madres del polen (CMP), en cada estadio de la meiosis.

\section{VIABILIDAD DE LOS GRANOS DE POLEN}

La viabilidad de los granos de polen se estimó con la solución de Müntzig, mezcla de carmín acético y glicerina en la propor-
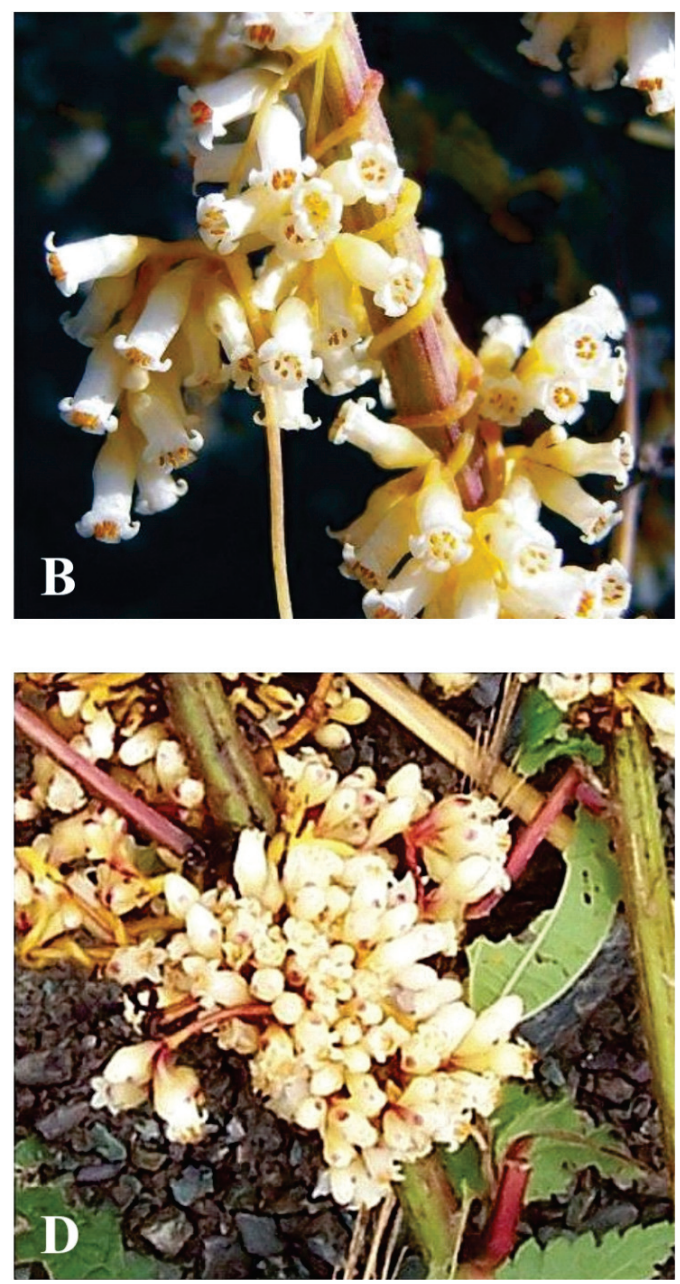

Fig. 1. A-D. Cuscuta parodiana. A) Sierra de Medina. B) Hualinchay (LIL 610027). C) Hualinchay (LIL 610030). D) Bárcena. 

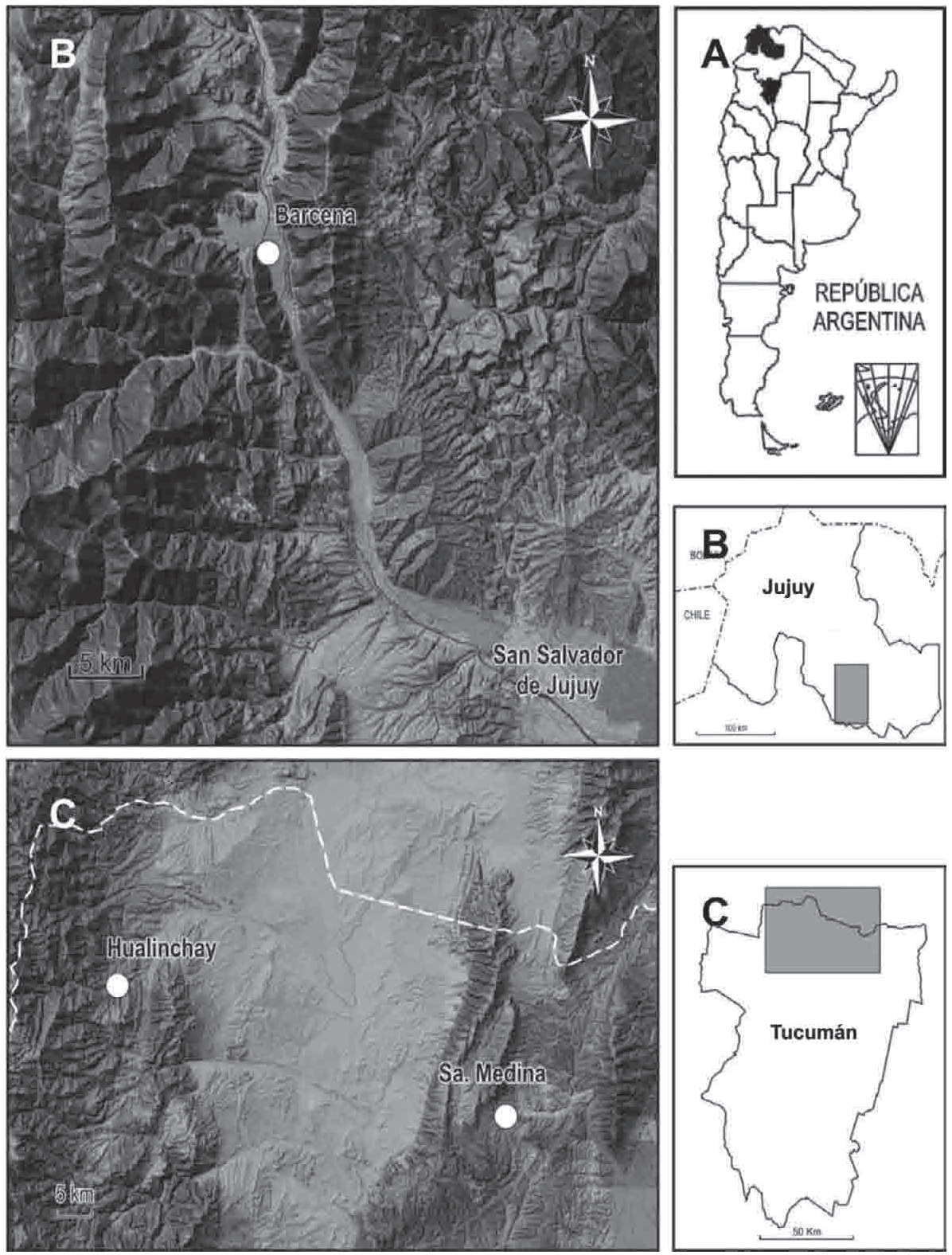

Fig. 2. A-C) Sitios de muestreo de Cuscuta parodiana. A) Región NOA. B) Provincia de Jujuy. C) Provincia de Tucumán.

ción de 1:1 (Sharma y Sharma, 1965). Se consideraron viables a los granos que presentaron coloración rojo intenso en su contenido celular y como no viables los granos carentes de tinción. Se tomó como estándar 1000 granos polen por taxón.

Los datos obtenidos durante el estudio de la meiosis y la viabilidad de los granos de polen se encuentran plasmados en la tabla 1.

Las microfotografías se tomaron con una video cámara Moticam 1000 (1.3 MP) acoplada a un microscopio Nikon Eclipse E-200, tanto para los análisis meióticos como para la viabilidad de polen. 
Tabla 1. Caracteres morfológicos florales, caracteres citológicos, formación de esporadas y viabilidad de polen expresada en porcentaje.

\begin{tabular}{|c|c|c|c|c|}
\hline \multicolumn{5}{|c|}{ CARACTERES MORFOLÓGICOS } \\
\hline CARACTER & Morfotipo I & Morfotipo II & Morfotipo III & Morfotipo IV \\
\hline Corola & Tubo corolino caliciforme & $\begin{array}{l}\text { Tubo corolino cilíndrico } \\
\text { de base angosta }\end{array}$ & Tubo corolino elipsoide & $\begin{array}{l}\text { Tubo corolino } \\
\text { subesférico }\end{array}$ \\
\hline Estilo & $\begin{array}{c}\text { Subsentado no sobrepasa la } \\
\text { altura del estigma }\end{array}$ & $\begin{array}{c}3 \text { veces la altura del } \\
\text { estigma }\end{array}$ & $\begin{array}{l}\text { De diferente tamaño, } \\
\text { el mas largo } 2 \text { veces la } \\
\text { altura del estigma }\end{array}$ & $\begin{array}{l}\text { Longitud } \\
\text { subiguales } \\
\text { relativamente } \\
\text { largos }\end{array}$ \\
\hline Ovario & Globoso & Semicircular & Ovoide & Esferoidal \\
\hline Escamas & $1 / 2$ fusionadas a la corola & $\begin{array}{l}2 / 3 \text { fusionadas a la } \\
\text { corola, escamas cortas } \\
\text { con pocas fimbrias }\end{array}$ & $\begin{array}{c}\text { la mitad inferior } \\
\text { fusionada a la corola } \\
\text { con fimbrias delgadas } \\
\text { y abundantes }\end{array}$ & $\begin{array}{l}2 / 3 \text { fusionados a } \\
\text { la corola }\end{array}$ \\
\hline \multicolumn{5}{|c|}{ Números cromosómicos, nivel de ploidia e irregularidades meióticas presentes en cada estadio } \\
\hline \multicolumn{5}{|c|}{ División I } \\
\hline $\mathrm{N}^{\circ}$ gamético & $\mathrm{n}=15$ & $n=30$ & $n=30$ & $\mathrm{n}=30$ \\
\hline $\begin{array}{l}\text { Nivel de } \\
\text { ploidía }\end{array}$ & Diploide & Tetraploide & Tetraploide & Tetraploide \\
\hline Profase I & tri o tetravalentes, citomixis & Diacinesis normal & Diacinesis normal & $\begin{array}{c}\text { Cromosomas en } \\
\text { diacinesis con } \\
\text { configuraciones } \\
\text { cuadrivalentes }\end{array}$ \\
\hline Metafase I & $\begin{array}{c}\text { Con cromosomas fuera de } \\
\text { placa, estructuras } \\
\text { cuatripartitas }\end{array}$ & $\begin{array}{l}\text { Con cromosomas fuera } \\
\text { de placa, agrupaciones } \\
\text { cromosómicas }\end{array}$ & $\begin{array}{l}\text { Cromosomas fuera de } \\
\text { placa }\end{array}$ & Normal \\
\hline Anafase I & Normal & $\begin{array}{l}\text { Puentes, cromosomas } \\
\text { con forma de } \mathrm{V} \text { invertida }\end{array}$ & Normal & Normal \\
\hline Telofase I & Citomixis & Citomixis & Normal & Normal \\
\hline \multicolumn{5}{|c|}{ División II } \\
\hline Metafase II & Normal & $\begin{array}{c}\text { Cromosomas fuera de } \\
\text { placa }\end{array}$ & $\begin{array}{l}\text { Husos acromáticos } \\
\text { perpendiculares }\end{array}$ & Normal \\
\hline Anafase II & Normal & $\begin{array}{l}\text { Puentes y cromosomas } \\
\text { rezagados }\end{array}$ & Normal & Normal \\
\hline Telofase II & Citomixis & Normal & Normal & Normal \\
\hline \multicolumn{5}{|c|}{ Formación de esporadas } \\
\hline & Normal & Citomixis & Normal & Normal \\
\hline \multicolumn{5}{|c|}{ Viabilidad de polen expresada en $\% n=1000$} \\
\hline $\begin{array}{l}\text { Granos } \\
\text { viables }\end{array}$ & $14 \%$ coloreados & $98 \%$ coloreados & $90 \%$ coloreados & $84 \%$ coloreados \\
\hline $\begin{array}{l}\text { Granos } \\
\text { inviables }\end{array}$ & $86 \%$ no coloreados & $2 \%$ no coloreados & $10 \%$ no coloreados & $\begin{array}{l}16 \% \text { no } \\
\text { coloreados }\end{array}$ \\
\hline
\end{tabular}

\section{RESULTADOS}

Los estudios morfológicos se realizaron en botones florales de cuatro morfotipos:

1) Morfotipo I (Población de Sierra de Medina, LIL 610028). Las flores de esta población se caracterizaban por poseer el tubo corolino caliciforme (Fig. 3A); escamas fimbriadas, la mitad de su longitud fusionada a la corola en la región basal (Fig. 3E). El gineceo es portador de un ovario globoso y estigma subsentado (Fig. 3I).

2) Morfotipo II (Población de Hualinchay, LIL 610027). En esta población, el tubo corolino es cilíndrico y de base angosta (Fig. 3B), escamas pequeñas que no superan la mitad de la longitud del tubo corolino, es- 

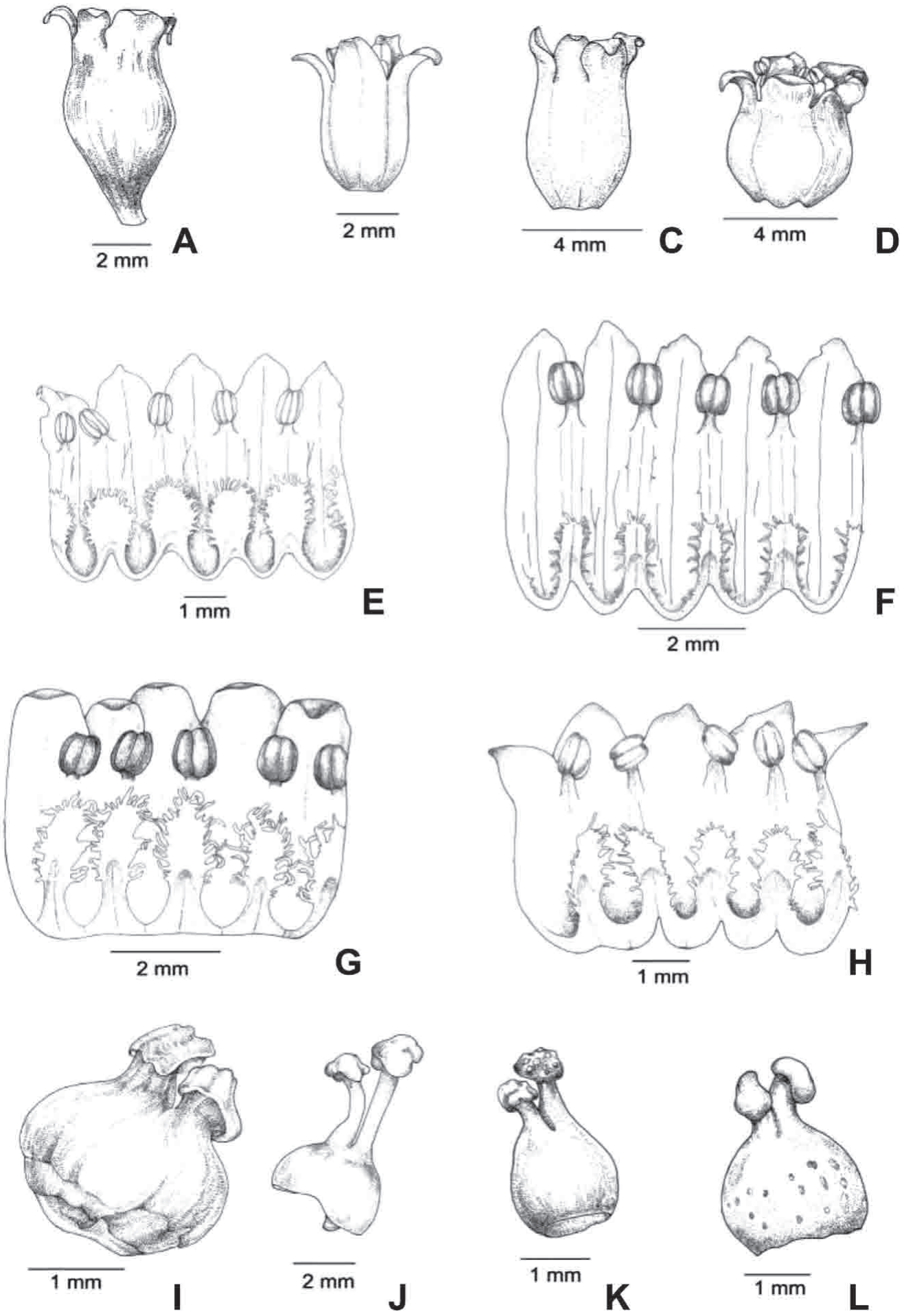

Fig. 3. Caracteres florales de Cuscuta parodiana. A, E, I) Morfotipo I. B, F, J) Morfotipo II. C, G, K) Morfotipo III. D, H, L] Morfotipo IV. 
casas fimbrias digitiformes con parte de su extensión soldadas a la corola (Fig. 3F). Es evidente en el gineceo un ovario ovoide y un estilo prolongado de aproximadamente tres veces la longitud del estigma (Fig. 3J).

3) Morfotipo III (Población de Hualinchay, LIL 610030). Las flores de esta población de Hualinchay tienen el tubo corolino elipsoide (Fig. 3C), escamas de igual o mayor a $2 / 3$ de la longitud del tubo, soldadas al éste en 1/3 de su longitud y con fimbrias (Fig. 3G).
Ovario semicircular; estilo no supera dos veces la longitud del estigma (Fig. 3K).

4) Morfotipo IV (Población de Barcena, LIL 610029). Tubo corolino subesférico (Fig. 3D) con escamas soldadas en la parte anterior de la corola, de $1 / 2$ del tamaño total de la longitud del tubo y con fimbrias (Fig. 3H). El gineceo con ovario esferoidal y estilo de aproximadamente dos veces la longitud del estigma (Fig. 3L).
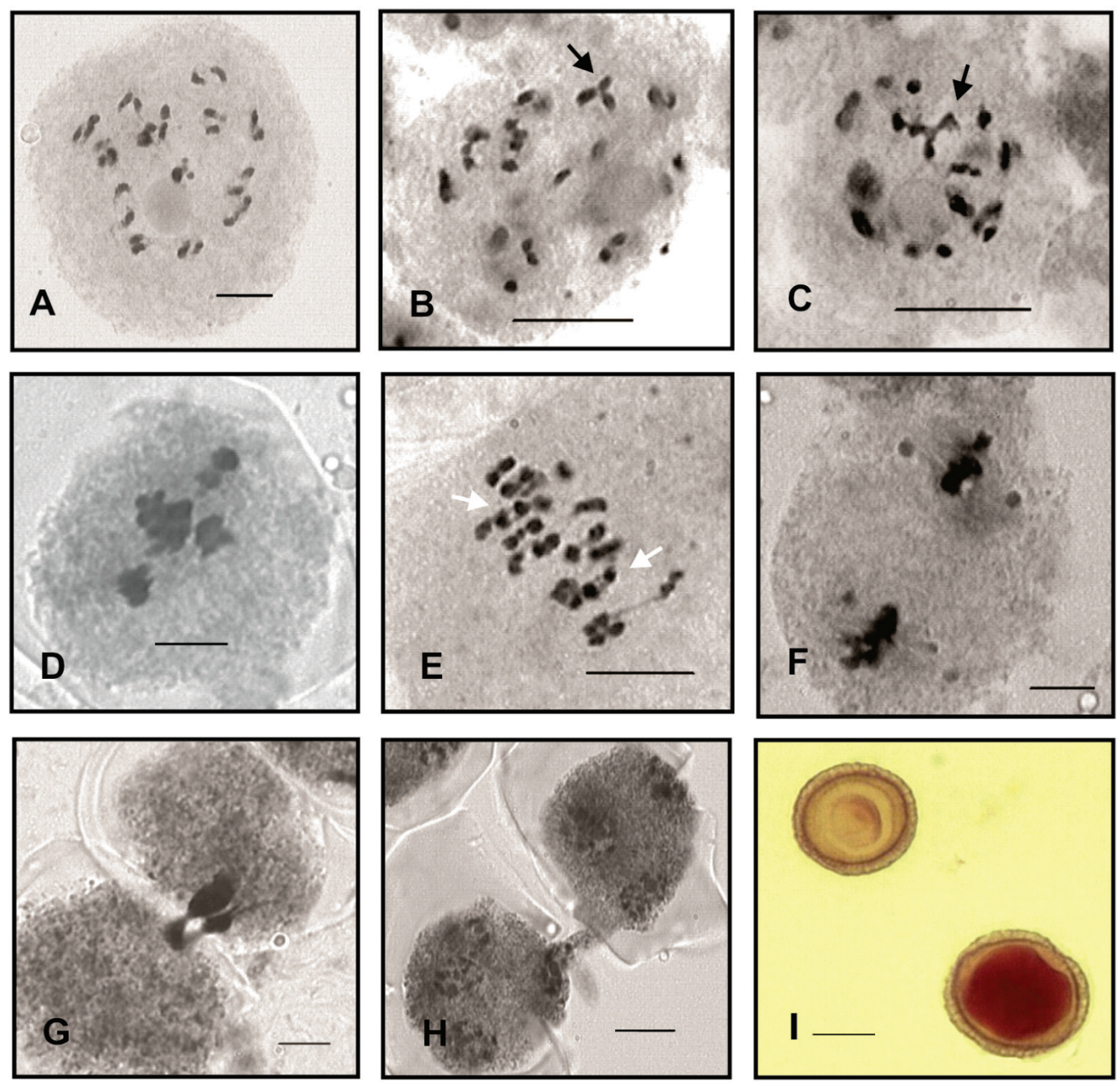

Fig. 4. Cuscuta parodiana. Morfotipo I. A) CMP en Diacinesis, $n=15$ y un nucléolo. B-C) CMP en diacinesis con presencia de 1 trivalente y 1 tetravalente respectivamente en forma de $Y$ [flechas negras]. D) MI con cromosomas fuera de placa. E) Inicio de Al con bivalentes con quiasmas terminales (flechas blancas). F) MII con cuerpos extranucleares. G) Citomixis en paquitene. H) Citomixis en TII. I] Granos de polen viable teñido y no viable traslúcido. Escala $=10 \mu \mathrm{m}$. 


\section{ANÁLISIS DE LA MEIOSIS}

Morfotipo I. El número haploide se evidenció en diacinesis con $n=15$ (Fig. 4A). Configuraciones de trivalentes y tetravalentes en forma de $\mathrm{Y}$ se observaron en esta etapa (Fig. 4B-C). En MI (Metafase I), 26,31\% de las células presentan cromosomas fuera de la placa ecuatorial (Fig. 4D); en AI (Anafase I) tempranas, se identifica claramente el inicio de la segregación de los cromosomas homólogos, con dos pares de bivalentes que se caracterizan por poseer quiasmas terminales (Fig. 4E). Las AI y TI (Telofase I) fueron regulares. En la segunda etapa de la división meiótica, el comportamiento de las células madres de polen (CMP) fue regular, con formación de esporadas normales. En ambas etapas de la meiosis se pusieron en evidencia cuerpos extranucleares débilmente coloreados, en número y tamaño variables (Fig. 4F). El fenómeno de citomixis se observó en ambas etapas de la división meiótica (Fig. 4G-H). La viabilidad de los granos de polen fue de un 14\% (Fig. 4I).

Morfotipo II. El número cromosómico gametofítico estimado en esta población fue $\mathrm{n}=$ 30. La MI fue regular (Fig. 5A), sólo 9\% de las células analizadas se presentaron con los cromosomas conformando tres grupos (Fig. 5BC) y cromosomas fuera de la placa metafásica (Fig. 5D). Las células en AI se caracterizaron por la presencia de puentes de cromatina en el $82 \%$ de ellas (Fig. 5E) y durante la segregación adquieren configuración en «V» invertida. (Fig. 5F-G). La citomixis se observa en TI en el 7\% de las células, este fenómeno ocurre a través de múltiples canales entre las células vecinas (Fig.5H). En metafase II (MII) el 3\% de las células presentaron 1-2 cromosomas fuera de la placa ecuatorial (Fig. 5I). Las AII se mostraron con segregación desbalanceada en el 4\% de ellas (Fig. 5J). En el 3\% de las tétradas también se observaron citomixis y la conexión se establece entre dos células unidas por un canal (Fig. 5K). Se estimó la viabilidad de los granos de polen en un 98 \% (Fig. 5L).

Morfotipo III. El número haploide de $\mathrm{n}=$ 30 se observó en diacinesis. La meiosis analizada en las CMP de esta población se mostró regular. No obstante, se destaca que el 1\% de las células en MI presentan cromosomas fuera de la placa ecuatorial (Fig. 6A) o en AI pueden encontrarse puentes de cromatina en el $2 \%$ de ellas (Fig. 6B) y cuerpos extranucleares múltiples en TI (Fig. 6C). En el 1\% de MII se presentaron con husos perpendiculares (Fig. 6D) y cromosomas fuera de la placa metafásica (Fig. 6E). Las esporadas observadas son regulares. El 90\% de los granos de polen analizados fueron viables (Fig. 6F).

Morfotipo IV. El número gametofítico encontrado en esta población fue $n=30$ (Fig. 7A). Al final de la prometafase los cromosomas adoptan configuraciones cuadrivalentes, con aspecto similar a las estructuras cuatripartitas observadas en especies con cromosomas holocinéticos (Fig. 7B). En AI los cromosomas rezagados se presentan en una frecuencia muy baja, sólo en el $2 \%$ de las células analizadas (Fig. 7C). La división II se observó regular, con formación de esporadas normales. En todos los estadíos fueron evidentes los cuerpos extranucleares (Fig. 7D-E). El $84 \%$ de los granos de polen fueron viables (Fig. 7F).

\section{DISCUSIÓN}

El análisis que Huzinker (1947) realizó en $C$. parodiana en base a los caracteres florales le permitieron reconocer un acentuado polimorfismo, por el cual distinguió las variedades $C$. parodiana var. typica y $C$. parodiana var. tucumana. Los caracteres evaluados por el autor tales como tamaño de los lóbulos de la corola, punto de unión de las escamas al tubo corolino y diámetro del anillo basal, no fueron constantes en las poblaciones estudiadas en este trabajo. Sin embargo, las diferencias en la forma y el tamaño del tubo corolino, así como de las escamas y la longitud del estilo, entre los cuatro morfotipos son persistentes y permiten identificarlos.

Al género Cuscuta se lo identifica como un complejo poliploide, con números cromosómicos que se extienden desde $2 \mathrm{n}=8$ - 60 . Los números base $\mathrm{x}=7,14$ y 15 son propuestos para los tres subgéneros (Fogelberg, 1938; Pazy y Plitmann, 1995). La observa- 

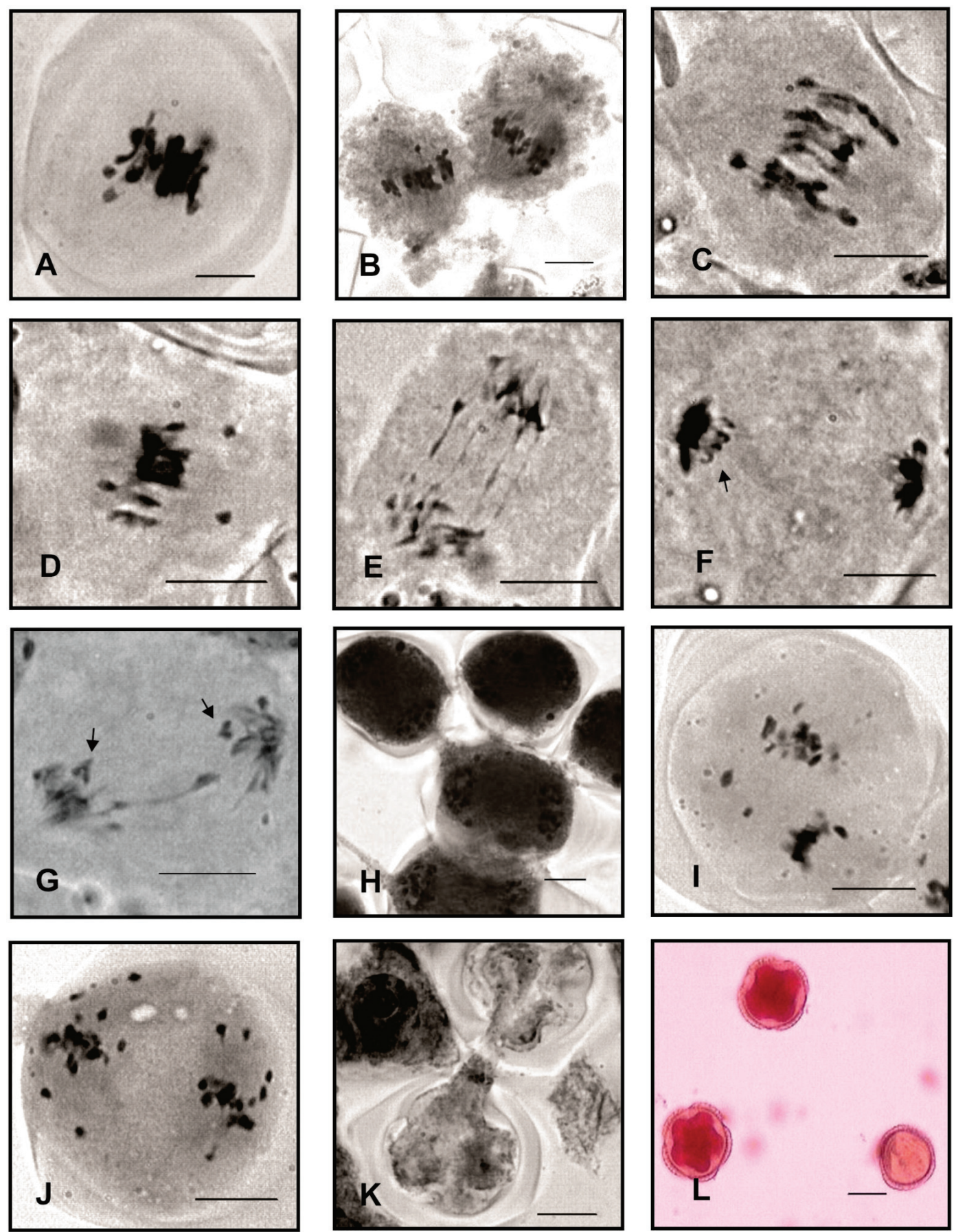

Fig. 5. Cuscuta parodiana. Morfotipo II. A) CMP en metafase I. B y C) Ml con cromosomas en tres grupos. D) Cromosomas fuera de placa en MI. E) Al temprana con puentes de cromatina. F y G] Disposición de los cromosomas en forma de $V$ invertida en Al (flechas). H) Citomixis en TI. I] Cromosomas fuera de placa en MII. J) All con segregación desbalanceada. KJ Citomixis en tétradas. L) Granos de polen viable e inviable. Escala $10 \mu \mathrm{m}$. 

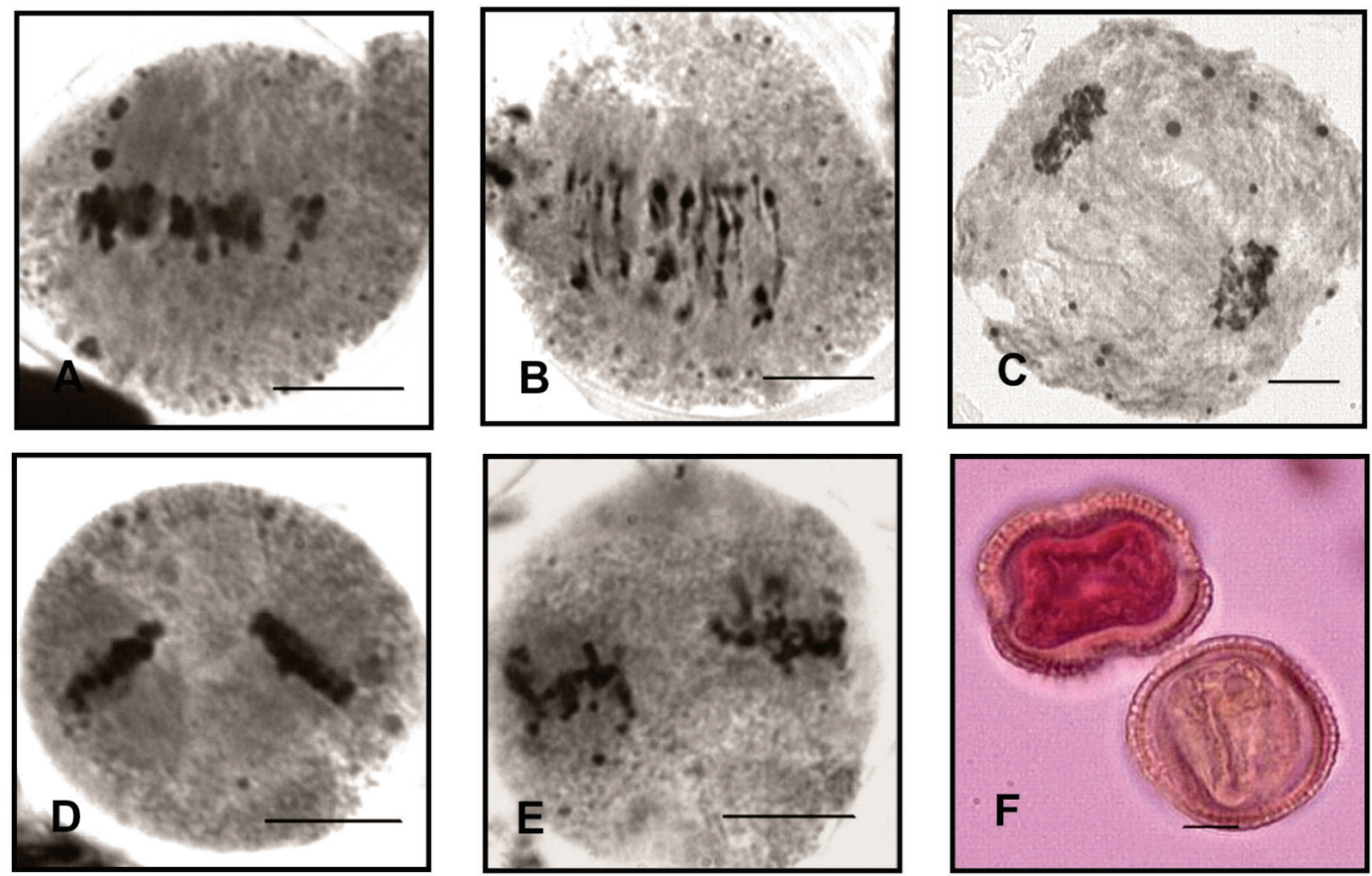

Fig. 6. Cuscuta parodiana. Morfotipo III. A] CMP en MI con cromosomas fuera de la placa. B] Puentes de cromatina en Al. C) Cuerpos extranucleares en AI.D) CMP en MII, con disposición perpendicular de los husos acromáticos. EJ CMP en MII con cromosomas fuera de placa. F) Grano de polen viable y grano no viable. Escala $=10 \mu \mathrm{m}$.

ción de ejemplares tetraploides y hexaploides en C. reflexa del subgénero Monogyna condujo a proponer otro número básico, $\mathrm{x}$ $=8$ (Kaul y Bhan, 1974, 1977).

Teniendo en cuenta que el número básico $\mathrm{x}=15$ es el más difundido en el género Cuscuta, podemos asumir que $C$. parodiana es una especie con dos citotipos, uno diploide con $\mathrm{n}=15$, presente en la población de Sierra de Medina y que concuerda con lo citado por Páez et al., (2011) y otro tetraploides $\mathrm{n}=30$ nunca antes observado, característico del resto de las accesiones analizadas en este trabajo. Estos últimos citotipos $2 \mathrm{n}=4 \mathrm{x}$ $=60$ se podrían haber originado mediante procesos de poliploidización semejantes a lo reportado para otras especies de Cuscuta (Fogelberg 1938; Kaul y Bhan, 1977; Lewis, 1980). Así las configuraciones de trivalentes y tetravalentes observadas durante MI en las poblaciones de Sierra de Medina y Barcena, indicarían una alta probabilidad de autopoliploidía (Folgelberg, 1938).
Asociaciones secundarias de cromosomas se presentaron en la población de Hualinchay (LIL 610027), sugiriéndose para este taxón un mecanismo semejante al mencionado por Mukherjee y Data (2006) en Ocimum spp., donde las asociaciones secundarias de cromosomas corresponderían a un poliploide secundario natural y que el taxón sufriría diploidización en el curso de la evolución mostrando comportamiento meiótico diploide.

Los antecedentes citogenéticos sostienen que solamente Cuscuta subgénero Cuscuta se caracteriza por desarrollar meiosis invertida (donde primeramente ocurre meiosis ecuacional y luego reduccional), la cual es frecuente en aquellos genomas con cromosomas holocinéticos. Por otra parte, para Cuscuta subgéneros Monogyna y Grammica, se citan cariotipos con cromosomas monocéntricos que, de hecho, se correlaciona con nuestras observaciones (Pazy, 1997; Pazy y Plitmann, 1991, 1994, 1995, 2002; García, 2001; García y Castroviejo, 2003). La presencia de las 

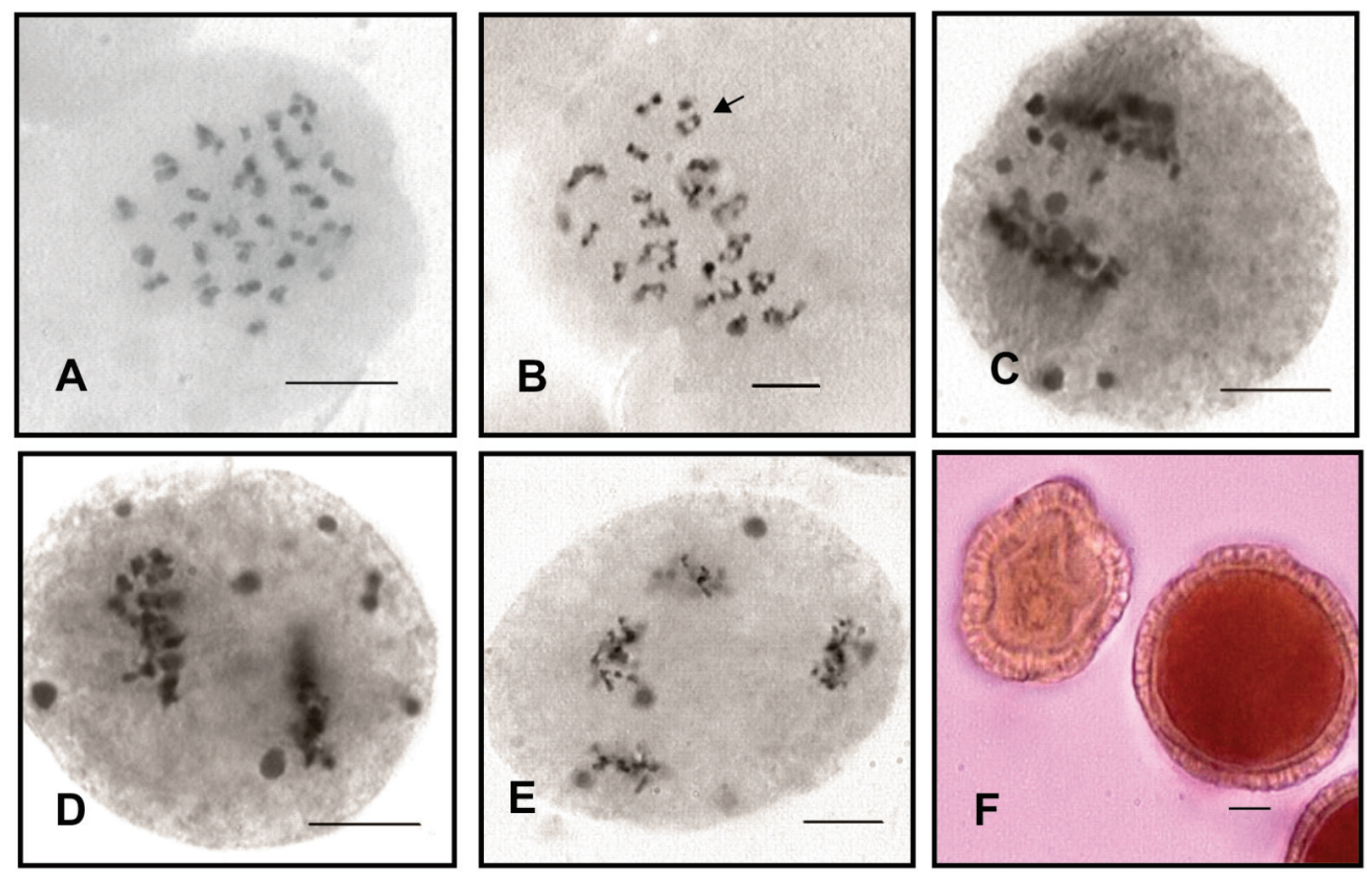

Fig. 7. Cuscuta parodiana. Morfotipo IV. A) Diacinesis $n=30$. B) CMP en diacinesis en vista polar con configuraciones cuadrivalentes (la flecha indica una de ellas en primer plano). C) Al con rezagados y cuerpos extranucleares respectivamente. D-E) MII y TII regulares con cuerpos extranucleares respectivamente. FJ Granos de polen viable e inviable. Escala $10 \mu \mathrm{m}$.

configuraciones cuatripartitas al comienzo de MI en la población de Bárcena, no puede homologarse con la morfología descripta del comportamiento de los cromosomas holocinéticos. En el presente estudio se asume que la cromatina adquiere una condensación diferencial durante la MI. La disposición de los cromosomas en «V» invertida durante AI, observada en la población de Hualinchay (Morfotipo II) pone en evidencia que la actividad cinética se concentra en más de una región de los cromosoma, tencionando a los husos desde los extremos (dos sitios) hacia un polo y traccionando la región media desde el polo opuesto. Como este taxón pertenece al subgénero Grammica, típico con cromosomas monocinéticos, este fenómeno podría ser explicado por la formación de trivalentes o tetravalentes. Esta condición no se pudo confirmar debido al reducido tamaño de los cromosomas. Además, la presencia de trivalentes o tetravalentes podría justificar los numerosos puentes de cromatina en AI.
Las poblaciones de Cuscuta parodiana analizadas hasta el momento conforman un complejo que presenta dos números cromosómicos. Las poblaciones con $\mathrm{n}=30 \mathrm{de}$ Bárcena y Hualinchay (LIL 610030) podrían tener un origen autopoliploide. A pesar de que la población de Sierra de Medina se considera diploide $(n=15)$ experimenta la presencia de configuraciones multivalentes, generalmente típicas de autopoliploides, semejante a las que presenta la población de Bárcena. Estas observaciones apoyan lo citado por autores precedentes quienes sugirieron que el número básico $\mathrm{x}=15$ podría haber surgido de un número de base ancestral $\mathrm{x}=7$ (Folgelberg, 1938; Lewis, 1980). Los puentes de cromatina, las asociaciones secundarias y las segregaciones desbalanceadas que presenta la población de Hualinchay (LIL 610027), hacen presumir que este taxón surgió por hibridación interespecífica de genomas por lo que se le inferiría que su origen fue por alopoliploidía. 
Los cuerpos extranucleares esféricos débilmente coloreados, de diferente tamaño y número, se presentan en todos los estadios de la meiosis en lo ejemplares de Sierra de Medina (Morfotipo I) y Bárcena (Morfotipo IV). Ellos son semejantes a los cuerpos extranucleares de C. cephalanthi Engelmann y C. gronovii (Fogelberg, 1938), visibles en el citoplasma cuando la membrana nuclear y el nucleolo se desorganizan al final de la primera etapa de la división meiótica, en profase. Ross (1981) mencionó la presencia de estos cuerpos en Cactaceae, de tinción negativa ante el reactivo de Feulgen, por lo que se puede inferir ausencia de ADN. Recientemente, Páez, Andrada, Ruiz de Bigliardo (2015) obtuvieron resultados similares en poblaciones del género Agalinis, donde los cuerpos extranucleares fueron DAPI negativo y NORs positivos sugiriendo presencia de proteínas similares a las asociadas al ARNr.

En dos poblaciones de C. parodiana (Morfotipo I y II), se observaron citomixis en la primera y segunda división meiótica, con formación de uno o más canales citoplasmáticos. Las causas de este fenómeno no se conocen con certeza, aunque los antecedentes indican que hay factores críticos en el proceso, como el estrés provocado por las condiciones adversas de temperatura, agentes mutagénicos, cambio en el nivel de ploidía o la hibridización. (Saraswathy Amma, Namboodiri, Panikkar, Sethuraj, 1990; Ghaffari, 2006; Sidorchuch, Deineko, Shumny, 2007; Kravets, 2011).

La viabilidad polínica disímil observada en Sierra Medina (14\%), frente a los mayores porcentajes $(98 \%, 90 \%$ y $84 \%)$ de las poblaciones restantes, podría asociarse sólo en parte a sus irregularidades meióticas, entre ellas la citomixis (23\%), el resto podría deberse a las condiciones ecofisiológicas del hospedante.

\section{CONCLUSIONES}

El estudio de los caracteres morfológicos y citológicos permitieron comparar las poblaciones analizadas. Podemos asumir de modo predictivo, que una revisión taxo- nómica minuciosa de Cuscuta parodiana y taxones afines, puede sentar las bases para comprender la naturaleza del polimorfismo que exhibe esta especie. Este polimorfismo también está reflejado a nivel citológico, ya que la especie presenta distintos citotipos y comportamientos meióticos en las poblaciones analizadas.

Esta es la primera vez que se registra en el subgénero Grammica actividad cinética en más de un punto de sus cromosomas, aunque se asume que presenta cromosomas monocinéticos. Grammica incluye la mayoría de los taxones y comprende las $3 / 4$ parte del total de las especies del género, están dispersos principalmente en América y carecen de estudios citogenéticos. Debido a ello, a medida que se realicen más análisis de este tipo se tendrá una mayor comprensión de los problemas inherentes al género, por tratarse del subgénero que sintetiza la complejidad de Cuscuta como un todo (Stefanovic et al., 2007).

\section{AGRADECIMIENTOS}

Se agradece al Geol. Alberto Gutiérrez y a la Lic. Lelia Bordón del Instituto de Iconografía de la Fundación Miguel Lillo por el diseño del mapa y realización de las láminas necesarias para este trabajo.

\section{BIBLIOGRAFÍA}

Fernandez, A. (1973). El ácido láctico como fijador cromosómico. Boletin de la Sociedad Argentina de Botánica 15 (2-3): 287-290.

Finn, V. V. (1937). Porivjal’na embriologija i kariologija dekil’koch vidiv Cuscuta. Journal of Institute of Botany, National Academy of Sciences of Ukranie, URSS 20: 83-105.

Fogelberg, S. 0. (1938). The cytology of Cuscuta. Bulletin of the Torrey Club 65: 631-645.

García, M. A. (2001). A new western Mediterranean species of Cuscuta (Concolvulaceae) confirms the presence of holocentric chromosomes in subgenus Cuscuta. Botanical Journal of the Linnean Society 135: 169-178. doi:10.1111/j.10958339.2001.tb01089.x 
García, M. A. y Castroviejo, S. (2003). Estudios citotaxonómicos en las especies ibéricas del género Cuscuta (Convolvulaceae). Anales del Jardín Botánico de Madrid 60 (1): 33-44.

Ghaffari, S. M. (2006). Occurrence of diploid and polyploid microspores in Sorghum bicolor (Poaceae) is the result of cytomixis. African Journal of Biotechnology 5 (16): 1450-1453.

Hunziker, A. T. (1947). Sinopsis de las especies argentino - uruguayas del género Cuscuta. Revista Argentina de Agronomía 4 (2): 123-147.

Kaul, M. L. H. y Bhan, A. K. (1974). Cytology of Cuscuta reflexa Roxb. Cytologia 39: 493-498. doi:10.1508/cytologia.39.493

Kaul, M. L. H. y Bhan, A. K. (1977). Cytogenetics of polyploids VI. Cytology of tetraploid and hexaploid Cuscuta reflexa Roxb. Cytologia 42: 125-136. doi:10.1508/cytologia.42.125

Kravets, E. (2011).The role of cell selection for pollen grain fertility after treatment of barley sprouts (Hordeum distichum L.) with UV-B irradiation. Acta Biologica Slovenica 54: 31-41.

Lewis, W. H. (1980). Polyploidy, Biological Relevance. Plenum Press, New York and London. (pp. 253-254).

Mukherjee, M. y Data, A. K. (2006). Secondary chromosome associations in Ocimum spp. Cytologia 71 (2): 149-152. doi:10.1508/cytologia.71.149

Páez, V. A., Andrada, A. R., Lozzia, M. E. y Toranzo, M. I. (2011). Estudios meióticos en cuatro espécies del género Cuscuta subgénero Grammica (Cuscutaceae). Lilloa 48 (1): 83-90.

Páez, V. A., Andrada A. R. y Ruíz de Bigliardo, G. E. [2015). Estudios meióticos en Agalinis fiebrigii y Agalinis genistifolia [Orobanchaceae]. Lilloa 52 (2): 154-160.

Pazy, B. (1997). Supernumerary chromosomes and their behaviour in meiosis of the holocentric Cuscuta babylonica Choisy. Botanical Journal of the Linnean Society 123: 173-176. doi:10.1111/ j. 1095-8339.1997.tb01411.x

Pazy, B. y Plitmann, U. (1991). Unusual chromosome separation in meiosis of Cuscuta L. Genome 34: 533-536.

Pazy, B. y Plitmann, U. (1994). Holocentric chromosome behaviour in Cuscuta (Cuscutaceae). Plant Systematic and Evolution 191: 105-109.

Pazy, B. y Plitmann, U. (1995). Chromosome divergence in the genus Cuscuta and its systematic implications. Caryologia 48 (2): 173-180.

Pazy, B. y Plitmann, U. (2002). New perspectives on the mechanisms of chromosome evolution in parasitic flowering plants. Botanical Journal of the Linnean Society 138: 117-122. doi:10.1046/ j.1095-8339.2002.00006.x

Ross, R. (1981). Chromosome counts, cytology, and reproduction in the Cactaceae. American Journal of Botany 68 (4): $463-470$.

Saraswathy Amma,C. K., Namboodiri, A. N., Panikkar, A. O. N. y Sethuraj, M. R. (1990). Radiation Induced Male Sterility in Hevea brasiliensis (Willd. ex Adr. De Juss.) Muell. Arg. Cytologia 55 (4): 547551.doi:10.1508/cytologia.55.547

Sharma, A. K. y Chatterji A. K. (1957). A cytological investigation of some Convolvulaceae as an aid in understanding their lines of evolution. Phyton 9: 143-157.

Sharma, A. K. y Sharma, A. (1965). Chromosome Techniques, Theory and Practice. Butteworth \& Co. (Publishers), London.( 474 pp].

Sidorchuk, Y. V., Deineko, E. V. y Shumny V. K. [2007). Peculiarities of cytomixis in pollen mother cells of transgenic tobacco plants (Nicotiana tabacum L.) with mutant phenotype. Cell Tissue Biology 1: 570-576.

Stefanovic, S., Kuzmina, M. y Costea, M. [2007). Delimitation of major lineages within Cuscuta subgenus Grammica (Convolvulaceae) using plastid and nuclear DNA sequences. American Journal of Botany 94 (4): 568-589. doi:10.3732/ ajb.94.4.568

Vasudevan, K. N. (1975). Contribution to the cytotaxonomy and cytogeography of the flora of the Western Himalayas (with an attempt to compare it with th flora of the Alps.] Part. I. Berichte der Schweizerischen Botanischen Gesellschaft 85: $57-84$

Vir Jee, D. U. y Kachroo, P. (1985). Chromosomal conspectus of some alpine-subalpine taxa Kashmir Himalaya. Chromosome Information Service 39: 33-35

Yuncker, T. G. (1932). The genus Cuscuta. Memoirs of the Torrey Botanical Club 18 (2): $113-323$.

Zuloaga, F. y Morrone, O. (1999). Cuscuta (Cuscutaceae). Catálogo de las plantas vasculares de la República Argentina II. Monographs in Systematic Botany. Missouri Botanical Garden Press. Vol II 74. 